\title{
Wallenberg Syndrome with Associated Motor Weakness in a Two-Week-Postpartum Female
}

\author{
David W. Louis ${ }^{a}$ Nimit Dholakia ${ }^{a} \quad$ Michael J. Raymond ${ }^{a, b}$ \\ ${ }^{a}$ The Commonwealth Medical College, Scranton, Pa., and ${ }^{b}$ Allied Services Integrated \\ Health System, Wilkes-Barre, Pa., USA
}

\section{Key Words}

Wallenberg syndrome $\cdot$ Lateral medullary infarct $\cdot$ Vertebral dissection $\cdot$ Dysphagia

\begin{abstract}
A 30-year-old, right-handed female presented 2 weeks postpartum with acute-onset severe headache, vertigo, and vomiting. Initial neurologic examination illustrated lingual dysarthria, horizontal nystagmus, right dysmetria on finger-to-nose testing, and weakness of the extremities. Magnetic resonance imaging showed a large, left lateral medullary infarction (Wallenberg syndrome) with cephalad extension into the ipsilateral pons as well as involvement of the left middle cerebellar peduncle. The patient was discharged 3 weeks later to an inpatient rehabilitation facility with gradual improvement of her symptoms. @ $2015 \mathrm{~s}$. Karger AG, Basel
\end{abstract}

\section{Introduction}

Lateral medullary syndrome (Wallenberg syndrome) is a well-known syndrome involving the posterior circulation of the medulla oblongata predominantly occurring secondary to atherosclerotic occlusion of the vertebral and basilar arteries, with rarer causes being embolism or dissection of the vessel $[1,2]$. There are a variety of 'Wallenberg plus' syndromes including Opalski syndrome that are aptly named for the addition of perplexing signs and symptoms, such as motor weakness or paralysis [3]. Additionally, documented cases have shown that pregnancy and the puerperium are risk factors for vertebral artery dissection leading to Wallenberg syndrome and its possible variants [4]. Here, we present an interesting combination of symptoms, neurological findings including motor weakness, and neuroimaging consistent with a variant of Wallenberg syndrome.

\section{KARGER 125


Louis et al.: Wallenberg Syndrome with Associated Motor Weakness in a Two-WeekPostpartum Female

\section{Case Report}

A 30-year-old, right-handed, G2P2 female with one previous normal vaginal delivery presented 2 weeks postpartum to the emergency department on 12/5/14 with complaints of significant headache, neck pain, vertigo, and vomiting. She gave a history of becoming dizzy after showering and hyperflexing her neck to dry her hair. She then walked into the bedroom and experienced several episodes of vomiting with left sided neck pain, headache, and paresthesia of the extremities. Emergency services were called when it was noted that her speech had become slurred. Past medical, surgical, and social history was noncontributory. Both pregnancies were complicated by mild gestational hypertension, for which pharmacologic treatment had not been required.

On presentation, her vitals were stable with a temperature of $98.4^{\circ} \mathrm{F}$, blood pressure of $132 / 102$, heart rate of $78 / \mathrm{min}$, respiratory rate of $20 / \mathrm{min}$, and oxygen saturation of $100 \%$ on room air. Her Glasgow Coma Scale Score (GCS) was 15. She was in acute distress and vomiting profusely. Neurologic assessment noted occasional hiccups, a weak cough, and obvious difficulties handling oral secretions. Her pupils were reactive bilaterally with the left being slightly more miotic. Horizontal nystagmus during primary gaze was worsened with left gaze. Her face appeared symmetric without facial droop. Mild-to-moderate lingual dysarthria was also observed. Strength in right and left upper extremities were both $4 / 5$ and right and left lower extremity strength was $4 / 5$ and $3 / 5$, respectively. Right handed finger-nose testing was positive for notable dysmetria. Abnormal laboratory results included a potassium of $2.9 \mathrm{mmol} / \mathrm{l}, \mathrm{CO}_{2}$ of $16 \mathrm{mmol} / \mathrm{l}$, glucose of $136 \mathrm{mg} / \mathrm{dl}$, and a positive anion gap of 17 $\mathrm{mmol} / \mathrm{l}$. Computerized tomography (CT) scan of the head was negative for an acute bleed, but computerized tomography angiography (CTA) of the head and neck indicated tapering and occlusion of the distal left vertebral artery at the level of C3, consistent with a left vertebral artery dissection. It was agreed by neurology to administer recombinant tissue plasminogen activator (alteplase), intubate due to worsening chest radiograph findings and possible aspiration of vomitus, and to transfer her to a tertiary care hospital to obtain a cerebral angiogram.

After transfer, repeat neurologic examination showed she was unable to move her eyes past the midline on the right. She withdrew to painful stimuli in all 4 extremities. Motor testing illustrated bilateral arm movements against gravity with right greater than left. Lower extremity movement with toe movement was present, but she could not move them against gravity. Ankle and knee jerks were both $0 / 4$ bilaterally. Due to the patient's history, physical, and recent imaging studies, magnetic resonance imagining (MRI) of the brain and magnetic resonance angiogram (MRA) of the head and neck were ordered showing a large left lateral medullary infarct with caudal extension near the cervical spinal cord and cephalad extension into the middle cerebellar peduncle. There were also multiple subcentimeter foci of T2 hyperintensity in the right inferior cerebellar hemisphere (fig. 1). MRA of the head and neck both showed loss of signal in the left vertebral artery consistent with slow flow or occlusion.

On 13/5/14, she was extubated, and routine CT status-post recombinant tissue plasminogen activator therapy showed a subtle subarachnoid hemorrhage. High-flow $\mathrm{O}_{2}$ was required after extubation to maintain oxygen saturation. Consultation with otolaryngology (ENT) and fiberoptic laryngoscopy showed left vocal cord paralysis with likely left lateral laryngopharyngeal dysfunction and poor cough/glottis clearance. It was decided to reintubate for airway protection. Later that day, she developed worsening left sided weakness. Neurology re-evaluated her, and repeat CT head was performed; however, no new infarctions or hemorrhage were noted. 
Louis et al.: Wallenberg Syndrome with Associated Motor Weakness in a Two-WeekPostpartum Female

On 15/5/14, general surgery performed a tracheostomy and placement of a percutaneous endoscopic gastrostomy tube. She was started on clopidogrel instead of warfarin secondary to risk of bleeding complications. Chest CT was performed due to concerns of tachycardia and oxygen desaturation which showed bilateral small pleural effusions with associated atelectasis.

The patient was discharged to an inpatient rehabilitation facility $(30 / 5 / 14)$ with a tracheostomy and percutaneous endoscopic gastrostomy tube in place, 11 days after initial presentation to commence intensive brain injury rehabilitation. She demonstrated gradual adaptive improvements and was discharged home on 22/7/14 under the supervision of her family. After discharge, she was scheduled for follow-up outpatient brain injury rehabilitation including comprehensive neuropsychological assessment.

Neuropsychological assessment was performed 5 months after infarction to determine the presence of any long-term deficits. Results indicate that despite normal scores on standardized neuropsychological indices (Halstead Impairment Index 0.3, GNDS 21), there was ongoing mild cognitive impairment. Primary deficits were observed in areas of sustained levels of attention/concentration, information processing speed, tactual localization memory, and nondominant left upper extremity sensorimotor abilities. In addition, adjustment difficulties including reactive depression, anxiety, and somatic preoccupation contributed to the clinical picture.

\section{Discussion}

Neurologic and functional recovery is dependent on the initial stroke severity [5]. The National Institutes of Health Stroke Scale (NIHSS) is commonly used as a method to determine patient recovery from ischemic stroke or those who may benefit from fibrinolytic agents such as tissue plasminogen activator. Studies have shown that the NIHSS has high sensitivity, specificity, and accuracy in determining patient prognosis and outcomes, even when taking the patient's age, gender, race, and history of previous stroke into consideration [6].

The incidence of stroke during pregnancy and in the postpartum period is low. However, recent studies indicate that the incidence of antenatal and postpartum hospitalizations for stroke has increased by 47 and 83\% from 1994-1995 to 2006-2007, respectively [7]. Risk factors for stroke during pregnancy most likely stem from the body's response to pregnancy, such as a state of hypervolemia, decreased systemic vascular resistance, increased cardiac output, and a state of hypercoagulability. Pathologic conditions such as chronic hypertension, gestational hypertension, preeclampsia-eclampsia, and tobacco use may also play a role in increasing the risk of stroke [8]. As reported above, our patient had a history of mild hypertension during both of her pregnancies. We can only speculate as to whether this was a precipitating factor regarding her postpartum ischemic stroke.

Lateral medullary syndrome (Wallenberg syndrome) is characterized by a specific constellation of symptoms. One of the most pathognomonic symptoms of Wallenberg syndrome includes pain and temperature sensory deficits of the ipsilateral face and contralateral extremities. This occurs as a result of damage to the spinal trigeminal tract and lateral spinothalamic tracts, respectively. Infarction of the vestibular nuclei may lead to vomiting, vertigo, and nystagmus, while injury to the inferior cerebellar peduncle may lead to ipsilateral ataxia. Ipsilateral pharyngeal, laryngeal, and palatal (such as dysphagia, hoarseness, and hiccups) ataxia types result from infarction of the nucleus ambiguous, the regulator of glossopharyngeal and efferent vagal reflexes. Lastly, Horner's syndrome with associated vague visual defi- 
Louis et al.: Wallenberg Syndrome with Associated Motor Weakness in a Two-WeekPostpartum Female

cits can results from damage to the ascending sympathetic fibers. The most common vessel involved in this presentation is the vertebral artery, as seen in this case, followed by the posterior inferior cerebellar artery $[1,2]$.

The fact that our patient had associated motor weakness indicates that this case cannot simply be labelled as Wallenberg syndrome. Review of the patient's MRI of the brain indicated that there was extension of the infarction superiorly to involve the ipsilateral pons via the left middle cerebellar peduncle as well as inferiorly to the lower aspects of the medulla, slow to the cervical spinal cord (fig. 1). The large extension of this lesion explains her unfortunate presentation. Her most significant motor weakness was noted in her left lower extremity. This most likely occurred as a result of involvement of the caudal medulla and possibly the cervical spinal cord, where motor fibers have already decussated in the medullary pyramid, allowing for ipsilateral symptoms relative to the location of the infarct. In addition, her 4/5 muscle strength in her other extremities may have been multifactorial in nature. Possible causes include the stress of just having undergone a stroke, undergoing multiple physical exams, and deconditioning secondary to her recent pregnancy. Lastly, we cannot rule out the possibility of motor fiber involvement superior to the main lesion leading to the mild contralateral weakness that was found on exam.

Finally, although our patient was not officially diagnosed as having a Wallenberg plus syndrome, these syndromes should be quickly reviewed, as knowledge of their presenting signs and symptoms can be invaluable to a patient's rapid diagnosis, treatment, and prognosis. Two major syndromes categorized as Wallenberg plus syndromes include Opalski syndrome (ipsilateral hemiparesis) and Babinski-Nageotte syndrome (contralateral hemiparesis) [9]. Their differences depend upon whether the original infarct is found to extend cranially or caudally in relation to the pyramidal decussation in the medulla, and has recently been the topic of debate. Liu et al. [10] considered the motor deficit to result from compromised medullary penetrating arteries which supply the pyramidal fibers below the decussation. Conversely, Dhamoon et al. [11] described this as a regional perfusion failure of the border zone between the anterior and posterior spinal arteries. In this argument, the likely cause would be a hemodynamic alteration due to vertebral artery occlusion, or in our case, dissection. There has been some debate on whether hyperreflexia should be included in the classic definition of Opalski syndrome. Hermann et al. [12] have pointed out that generalized hyperreflexia and/or a Babinski's sign rarely present in these patients since Opalski's original description [13].

\section{Conclusions}

Lateral medullary stroke (Wallenberg syndrome) can have a variable presentation of signs and symptoms dependent on the brainstem nuclei and spinal tracts involved. Diagnosis of these syndromes requires clinical skill to determine neurologic signs and symptoms as well as radiologic studies including MRI/MRA of the head and neck to identify the location of the lesion. The prognosis of a stroke can be determined by its severity and results of serial neuropsychological assessment.

\section{Statement of Ethics}

The authors have no ethical conflicts to disclose. 


\section{Case Reports in Neurology}

\begin{tabular}{l|l}
\hline \multicolumn{2}{l|}{ Case Rep Neurol 2015;7:186-190 } \\
\hline DOI: $10.1159 / 000440712$ & $\begin{array}{l}\text { C } 2015 \text { S. Karger AG, Basel } \\
\text { www.karger.com/crn }\end{array}$ \\
\hline
\end{tabular}

Louis et al.: Wallenberg Syndrome with Associated Motor Weakness in a Two-WeekPostpartum Female

\section{References}

1 Pearce JMS: Wallenberg's syndrome. J Neurol Neurosurg Psychiatry 2000;68:570.

12 Wilkins R, Brody I: Wallenberg's syndrome. Arch Neurol 1970;22:379.

-3 Parathan KK, Kannan R, Chitrambalam P, Senthil KA, Deepthi N: A rare variant of Wallenberg's syndrome: Opalski syndrome. J Clin Diagn Res 2014;8:MD05-MD06.

4 Levy M, Levy E, Maimon S: Atypical postpartum stroke presenting as Opalski syndrome: case report and review of the literature. Case Rep Neurol 2011;3:191-198.

$\checkmark 5$ Jørgensen HS, Nakayama H, Raaschou HO, Olsen TS: Stroke. Neurologic and functional recovery the Copenhagen Stroke Study. Phys Med Rehabil Clin North Am 1999;10:887-906.

-6 Adams HP, Woolson RF, Clarke WR, Davis PH, Bendixen BH, Love BB, Wasek PA, Grimsman KJ: Design of the Trial of Org 10172 in Acute Stroke Treatment (TOAST). Control Clin Trials 1997;18:358-377.

-7 Grear KE, Bushnell CD: Stroke and pregnancy: clinical presentation, evaluation, treatment, and epidemiology. Clin Obstet Gynecol 2013;56:350-359.

-8 Feske SK: Stroke in pregnancy. Semin Neurol 2007;27:442-452.

-9 Pandey S, Batla A: Opalski's syndrome: a rare variant of lateral medullary syndrome. J Neurosci Rural Pract. 2013;4:102-104.

$>10$ Liu CY, Chang FC, Hu HH, Hsu LC: Ipsilateral crural monoparesis in lateral medullary infarction due to vertebral artery dissection. Eur J Neurol 2006;13:e8-e9.

11 Dhamoon SK, Iqbal J, Collins GH: Ipsilateral hemiplegia and the Wallenberg syndrome. Arch Neurol 1984;41:179-180.

-12 Hermann DM, Jung HH, Bassetti CL: Lateral medullary infarct with alternating and dissociated sensorimotor deficits: Opalski's syndrome revisited. Eur J Neurol 2009;16:e72-e74.

-13 Sanahuja J, Begue R, García-Cerecedo T: Ischemic infarction in the territory of the posteroinferior cerebellar artery with spinal cord involvement (Opalski syndrome). Neurol Barc Spain 2003;18:117-118.
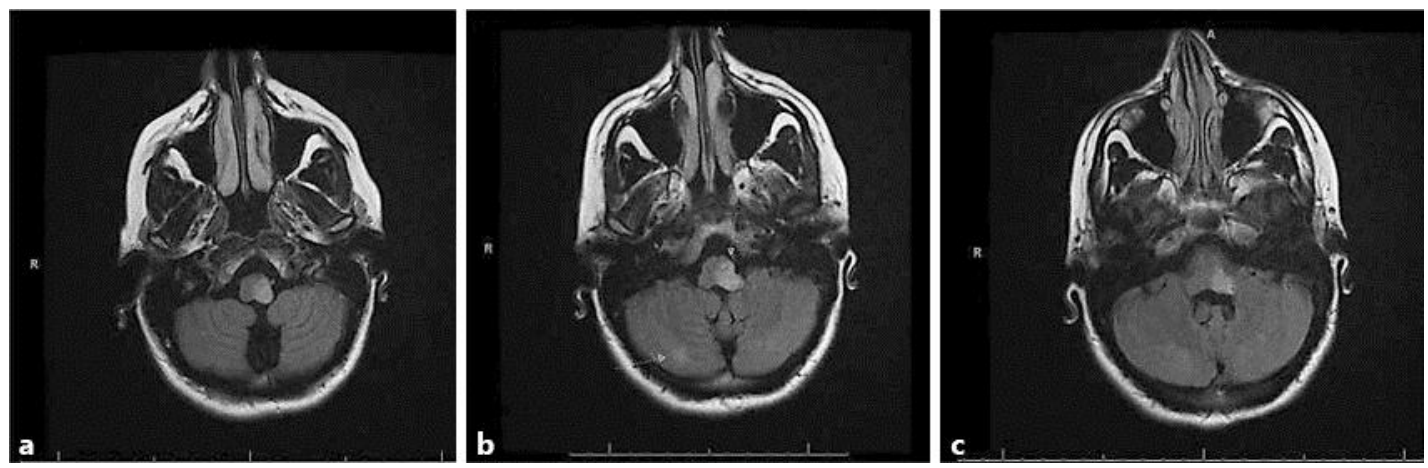

Fig. 1. MRI of the brain revealing a large, left-sided infarction extending inferiorly to the lower medulla (a) and superiorly to the left middle cerebellar peduncle (c) on T2-weighted images. A small area of hyperintensity can be seen in the right lobe of the cerebellum. 